\title{
Caminando hacia una profesión globalizada. El proceso de adopción de las Normas Internacionales de Información Financiera (NIIF) del IASB en Argentina
}

\author{
Juan Pablo Romano Pastor \\ Universidad del Aconcagua - Mendoza - Argentina.
}

\begin{abstract}
Resumen
En el marco del profundo cambio de paradigmas que la profesión contable llevó adelante desde mediados del siglo pasado (desde el paradigma patrimonialista hacia el paradigma de la utilidad), la profesión argentina inició en el año 1998 el proceso de adaptación (primeramente) y finalmente adopción de las Normas Internacionales de Información Financiera, el cual se concretó en el año 2010 con la sanción de la Resolución Técnica $N^{\circ} 26$. Durante esos años, se sucedieron diferentes etapas que marcaron un profundo cambio en la forma de "pensar" y de "hacer" contabilidad. El resultado: nuevos desafíos para los profesionales y la necesidad de percibir al contador público como un profesional de clase mundial. Partiendo de estos cambios, se analiza cada una de las fases que se llevaron adelante para concluir en la obligatoriedad de utilizar IFRS en la preparación de información financiera para cierto tipo de entes en la República Argentina.
\end{abstract}

\section{Palabras clave}

Adopción; integración; paradigma; NIIF; obligación.

\begin{abstract}
In the frame of the deep change of paradigms that the countable profession took forward from middle of last century, (from the paradigm "patrimonialist" towards the paradigm of the "usefulness" (utility)), the Argentine profession initiated in the year 1998 the process of adjustment (first) and finally adoption of the International Financial Reporting Standards, which made concrete in
\end{abstract}

Recibido: 8-06-2012 - Versión final aceptada: 19-10-2012 
the year 2010 with the sanction of the Technical Resolution $\mathrm{N}^{\circ} 26$. During these years, there happened different stages that marked a deep change in the way of "thinking" and of "doing" accounting. The result: new challenges for the professionals and the need to develop the CPA as a professional of world class. Departing from these changes, it is analyzed each of the phases that removed forward to conclude in the obligatory nature to use IFRS in the preparation of financial information for certain type of entities in Argentina.

\section{Key words}

Adoption; integration; paradigm; IFRS; obligation.

\section{Introducción}

Sin lugar a dudas tiempos agitados definen la actualidad de la profesión contable en el mundo. Términos como incertidumbre, integración, paradigmas, se hacen eco de una realidad que parecía convertirse rutinaria en una profesión sin sobresaltos. Podemos decir, hoy en día, que somos testigos de los cambios más trascendentales que sufre la profesión contable desde su aparición y consolidación (entre los siglos XI y XVI). ¿Se produce un cambio en la mecánica de registración? No. La partida doble sigue en plena vigencia. ¿Entonces? Existe un replanteo en los por qué y para qué de la disciplina. Replanteo que implica un profundo cambio de paradigmas, entendiéndose a éstos como todas aquellas prácticas que definen el objeto de una disciplina científica en un lapso determinado de tiempo.

Estas profundas modificaciones nos plantean la necesaria reflexión sobre una realidad paradigmática que se traduce en un cambio de enfoque radical respecto del objeto de hacer contabilidad. Podemos afirmar que hasta el año 1930 nuestra disciplina se estructuró alrededor del enfoque patrimonialista, el cual otorgaba prioridad fundamental, como objeto de los estados financieros, a lograr que el propietario del ente conociera la situación patrimonial y económica del mismo en una fecha determinada. En consecuencia, la visión se completaba en un estricto control patrimonial, con preeminencia de su mantención.

A partir del derrumbe de los mercados durante la crisis de 1930, comienza el marcado cambio de paradigma hacia un enfoque de la utilidad, el cual ve a la contabilidad financiera como el medio facilitador 
para la toma de decisiones de los usuarios. Esta novedad implicó la estructuración de la disciplina contable alrededor de los mercados de capitales y de los proveedores, no sólo de capital, sino de crédito, de inversión, etc. Se le otorgó una mayor importancia a lo financiero, lo cual se tradujo, entre otras cosas, en la aparición de modelos de estados de fuentes y aplicaciones de fondos y en la denominación de los estados contables como "estados financieros", denominación que las normas internacionales conservan hasta hoy.

Todas estas modificaciones plantean hoy en día un nuevo escenario contable internacional, basado fundamentalmente en cinco pilares:

- Supremacía de la contabilidad anglosajona

- Esencialidad sobre prudencia

- Contabilidad a valor razonable (fair value accounting)

- Internacionalización de la regulación contable

- Principios versus reglas

\section{Supremacía de la contabilidad anglosajona}

Este pilar se traduce en el paulatino abandono de los denominados "principios de contabilidad generalmente aceptados" o PCGA, en beneficio de una contabilidad orientada a reglas, con micro-orientación de los negocios y una fuerte presencia del juicio profesional a la hora de realizar interpretaciones de hechos contables concretos. En forma concomitante al enfoque de la utilidad, hay una acentuada orientación al inversionista como usuario principal de los estados financieros.

\section{Esencialidad sobre prudencia}

Hablar de esencialidad implica dar prioridad al principio de la realidad económica por sobre la forma jurídica que adopte el hecho o, dicho de otro modo, "sustancia sobre forma". Esto se traduce, por ejemplo, en la definición de los activos de acuerdo al control que se tenga sobre los beneficios económicos futuros que generen y no sobre la propiedad del mismo (fundamento de la contabilización de arrendamientos financieros de NIC 17). De la misma forma, la segregación de componentes financieros implícitos y la consolidación basada en el control, fuera de 
cualquier requisito formal, coadyuvan a reforzar la idea de que la esencia económica prima por sobre la forma jurídica del negocio.

\section{Contabilidad a valor razonable (fair value accounting)}

La tendencia de las normas emitidas por los distintos organismos internacionales es redireccionar los métodos de medición de los elementos de los estados contables hacia el fair value accounting o contabilidad a valor razonable. Esto es utilizar el precio que sería recibido por vender un activo o pagado por transferir un pasivo en una transacción ordenada entre participantes de mercado a la fecha de medición, independientemente si el precio es observable directamente o estimado por técnicas de valoración. La emisión en 2011 de la NIIF 13, ha facilitado una definición de lo que es el valor razonable (definido con anterioridad de forma ambigua) y establecer escalas de criterios para poder obtener una medición más fiable.

\section{Internacionalización de la regulación contable}

La aparición en 1973 del International Accounting Standard Committe (IASC - Comité de Contabilidad escindido del ICCAP) como primer organismo emisor de normas contables internacionales y en 1977 de la International Federation of Accountants (IFAC), representan los dos hitos iniciales de la globalización de la profesión contable. La tendencia adoptada de dejar fuera del ámbito de la profesión la emisión de las normas contables, es corolario de una realidad que prima en el mundo en la búsqueda de normas contables objetivas y de alta calidad.

Actualmente el International Accounting Standard Board (IASB), organismo técnico de la IFRS Foundation, es el ente emisor por excelencia de normas internacionales de información financiera (IFRS o NIIF).

\section{Principios versus reglas}

El debate radica en identificar cuál es el grado de detalle que debiera tener un juego de estándares internacionales para la preparación de información financiera con el objeto que ésta sea de alta calidad y útil para los agentes intervinientes en los mercados de capitales. El conflicto comienza 
cuando encontramos emisores que basan sus juegos de normas en principios generales (PCGA) y otros que los basan en reglas (ej. FASB y sus US GAAPs). Debemos considerar que las crisis derivadas de las caídas de Enron y Worldcom plantearon una fuerte crítica hacia los emisores que basaron sus estándares en reglas, dado que estos hechos delataron la fragilidad de sus normas. No obstante esto, consideramos que el actual proceso de convergencia entre IASB y FASB debiera completarse en el largo plazo con la emisión de un único juego de estándares global (Global GAAPs).

\section{¿Cuál será el probable escenario contable mundial?}

El juego de todos estos pilares descritos incide sobremanera a la hora de intentar una estimación o una mera aproximación hacia el futuro panorama normativo de la contabilidad en el mundo. Dejaremos fuera de discusión la posibilidad de desarrollo futuro de Global GAAPs a través del trabajo común entre el IASB y el FASB. Consideraremos solamente el proceso de convergencia iniciado.

En estas condiciones, existe cierto consenso en teorizar que la estructura mundial de las normas se centrará en la adopción de las Normas Internacionales de Información Financiera (NIIF) del IASB, de acuerdo al siguiente detalle:

- NIIF completas: adoptadas en forma obligatoria para entes de interés público y como opción para el resto de los entes.

- NIIF para pymes: adoptadas en forma obligatoria para el resto de los entes, excepto microempresas, las cuales tendrán la opción de adoptarlas.

- Norma contable local: aplicable en forma obligatoria para microempresas, quienes a su vez tendrán la opción de aplicar NIIF completas o NIIF para pymes.

De este detalle anterior, advertimos que los organismos emisores de normas contables profesionales de cada país trabajarán sobre el desarrollo de una norma contable nacional, más simplificada que las NIIF para pymes, a ser aplicadas por aquellas microempresas que no hayan adoptado como opción estas últimas o las NIIF completas. El siguiente gráfico ilustra la situación desde el punto de vista del tipo de ente. 


\begin{tabular}{|c|c|c|}
\hline Tipo de ente & $\begin{array}{c}\text { Aplicación } \\
\text { obligatoria }\end{array}$ & $\begin{array}{c}\text { Aplicación } \\
\text { opcional }\end{array}$ \\
\hline Entes de interés público & NIIF completas & --------------- \\
\hline $\begin{array}{c}\text { Resto de los entes, } \\
\text { excepto microempresas }\end{array}$ & NIIF para pymes & NIIF completas \\
\hline Microempresas & Norma contable local & $\begin{array}{c}\text { NIIF completas } \\
\text { NIIF para pymes }\end{array}$ \\
\hline
\end{tabular}

Fuente: elaboración propia.

\section{Implementación de NIIF en el mundo: adoptar, adaptar, ajustar}

Al momento de decidir sobre la implementación de los estándares internacionales como norma obligatoria para la preparación de los estados financieros, podemos advertir tres formas diferentes de actuar. Una primera opción es adopción plena para todos los entes que presenten estados financieros auditados, opción que implica la instauración inmediata y sin transición de NIIF, llevado adelante por países como Uruguay, Panamá, Costa Rica, Perú, Chile y Venezuela. Las ventajas en este modo radican en la implementación inmediata y rápida inserción internacional. Lógicamente, las desventajas radican en la falta de transición hacia el modelo IASB, el brusco abandono de modelos contables vigentes en cada país y la falta de la debida preparación por parte de los profesionales que encabezaron los procesos de adopción de los nuevos modelos contables en cada empresa.

La segunda alternativa es la de adaptación a normas locales. Este modelo fue llevado adelante por la profesión argentina en una primera etapa, como veremos luego. La ventaja radica en el logro de un cambio paulatino y ordenado en las prácticas y modelos contables y en la posibilidad de lograr un afianzamiento en torno a una norma contable intermedia que no es ni $100 \%$ NIIF ni $100 \%$ norma local (modelo híbrido).

Por último, el tercer modo, llevado adelante, entre otros, por la Unión Europea, México y Argentina (en una segunda etapa), es el de adopción plena para entes cotizantes y/o de interés público y optativo 
para el resto de los entes. Este modelo cumple en parte con el propósito general del IOSCO (organismo que agrupa bolsas de valores y mercados financieros de todo el mundo) de facilitar la comparabilidad de los estados financieros para ser analizados por inversores actuales y potenciales; y, por otro lado, permite (en el caso argentino) optar al resto de los entes por su aplicación, en su versión full o para pymes. Como arista interesante podemos agregar que aquellos entes que no fueran obligados directamente por norma a aplicar NIIF completas y quieran acceder a préstamos internacionales, deberán presentar estados financieros bajo NIIF completas. La principal desventaja en el modelo radica en la imposibilidad de efectuar comparación interempresas, sean cotizantes o de interés público o no, y la coexistencia de distintos juegos de normas contables obligatorias de acuerdo con el tipo de entes que se trate.

\section{El caso argentino}

Antes de comenzar a analizar el modo por el cual Argentina se insertó en el contexto internacional NIIF, realizaremos una breve reseña sobre cómo está organizada la profesión contable argentina.

La ley No 20488, de ejercicio profesional, define los ámbitos de actuación y las facultades reservadas para el uso del título de "contador público", el cual sólo podrá ser ejercido por personas de existencia visible. Esta misma norma determina que para que un egresado de cualquier universidad (pública o privada) quede habilitado para el ejercicio profesional, debe matricularse en el Consejo Profesional respectivo de la jurisdicción en la que vaya a ejercer. En el caso argentino, existen 24 Consejos Profesionales, uno por cada provincia y uno más actuante en el ámbito de la Ciudad Autónoma de Buenos Aires. Además de llevar registros de matrículas y velar por el ejercicio ético de la profesión, estos Consejos tienen la potestad de dictar normas profesionales contables, de auditoría, de sindicatura y de ética.

Los Consejos, a su vez, se encuentran agrupados en una institución de segundo grado, que es la Federación Argentina de Consejos Profesionales de Ciencias Económicas (FACPCE), cuyo ente técnico (CENCyA - Consejo Emisor de Normas de Contabilidad y Auditoría) es quien propone las normas técnicas que serán aprobadas (o no) por cada Consejo Profesional. Esta potestad de aprobación, modificación 
o derogación de normas técnicas reservadas, llevó a que se suscribiera el Acta de Catamarca en el año 2002, por la cual se formalizó el compromiso por parte de los Consejos Profesionales de adoptar las normas técnicas tal cual fueran propuestas por FACPCE. Esta situación, institucionalizada a través de la Resolución No 242/2003, logró la armonización de las normas contables profesionales en la República Argentina.

Una vez analizada la estructura, pasamos a detallar cuáles fueron los pasos que dio la profesión argentina en su proceso de inserción internacional, iniciado en el año 1995 con la incorporación de la FACPCE a la IFAC.

\section{Primer momento: adaptación de normas}

El primer paso, cuyo origen fue la incorporación a la IFAC y la necesidad de cumplimiento de las Statements of Membership Obligations (SMO), se inició en el año 1998 con los primeros proyectos de normas contables profesionales "adaptadas" a los modelos de normas internacionales vigentes. Esto culminó en diciembre del año 2000 con la aparición de las Resoluciones Técnicas (RTs) 16 a 19, las cuáles representaron un paso muy importante hacia el ámbito internacional. La profesión contable argentina, por ejemplo, lograba tener por primera vez un marco conceptual propio (RT 16), que si bien no era el mismo, se basaba -en líneas generales-, en el Conceptual Framework de 1989 del IASC. Los avances más importantes hacia normas internacionales se condensaron en:

- Mantención de estados financieros sin reexpresar en contextos de estabilidad monetaria (moneda nominal)

- Reexpresión sólo en casos de inestabilidad monetaria definida ésta por FACPCE. La decisión del contexto difiere del caso de NIC 29 (hiperinflación)

- Medición de activos y pasivos teniendo en cuenta el destino más probable. Para aquellos destinados a la venta, negociación, cobranza anticipada, cancelación anticipada, utiliza fair value según diferentes modalidades. Para aquellos destinados a uso, cobro o pago al vencimiento y otros destinos, valores históricos bajo la forma de costo amortizado, costo incurrido o valor nominal. 
Caminando hacia una profesión globalizada. El proceso de adopción de las Normas ...

Debemos destacar que para este primer paso de convergencia, las normas utilizadas fueron todas, con excepción de la NIC 1, NIC 19, NIC 20, NIC 26 y NIC 30. De acuerdo con la fecha en que se desarrolló esta convergencia, la versión de las "standards" fue la misma que adoptó la Unión Europea mediante el Reglamento No $1725 / 2003$. Es importante agregar que el resto de las normas fueron adaptadas con algunas diferencias, las cuáles fueron identificadas en los anexos que acompañaban cada una de esas normas. Algunos ejemplos eran:

- NIC 2 no admite fair value para inventarios y en Argentina eran valuados a costo de reposición.

- Respecto a los pasivos contingentes, en Argentina se requiere alta probabilidad de ocurrencia para su contabilización.

- Referido específicamente a NIC 29, la reexpresión que define la RT 6 es exigida en inflación y no solamente en hiperinflación.

\section{Segundo momento: consideración de NIIF como normas supletorias}

En el año 2005 se produjo una modificación al punto 9 de la segunda parte de la RT 17 a través de la Resolución № 312/2005. De acuerdo con esta nueva redacción, cuando una cuestión no prevista no hubiere podido ser resuelta de acuerdo con las reglas de medición contable en particular, deberá buscarse solución a través de:

- Reglas de medición contable, en general, o

- definiciones contenidas en el marco conceptual argentino (RT 16), o

- en las reglas derivadas de NIIF, IFRIC, NIC y SIC

Como vemos, esta segunda etapa comenzó a considerar las normas internacionales como norma técnica supletoria para los casos que una situación en particular no pudiera ser resuelta a través de las normas contables argentinas. En esta circunstancia influyeron también sobremanera las constantes y crecientes modificaciones que el IASB comenzó a realizar sobre el cuerpo de normas base que sirvió 
para la sanción de las RT 16 a 19. Por tanto, estas normas comenzaron a quedar desactualizadas respecto de los estándares utilizados para su redacción.

\section{Tercer momento: adopción de las NIIF}

Un tercer momento, y el más trascendental, sucedió a partir de marzo de 2009, cuando la RT 26 adopta las normas internacionales de información financiera (NIIF) en un sentido amplio como normas obligatorias para ciertos entes y optativas para el resto. La obligatoriedad genérica abarca a los entes que cotizan (tanto por acciones como por emisión de deuda), con exclusiones. Con posterioridad esta RT 26 fue modificada por RT 29 para incluir, entre otras modificaciones, la posibilidad de optar, para entes no obligados, por las NIIF para pymes.

\section{Cuarto momento: adopción como normas supletorias de normas de otros organismos emisores con un marco conceptual similar}

El último avance se logró a partir del Proyecto de Resolución Técnica No 18, que fue convertido en Resolución Técnica No 30 el 2 de mayo de 2011. A partir de este nuevo pronunciamiento, se agrega la posibilidad de considerar como norma supletoria a todos los pronunciamientos de otros emisores de normas contables en tanto y en cuanto posean un marco conceptual similar al que tiene adoptado la profesión argentina en la RT 16.

\section{Análisis particular de la adopción de NIIF en Argentina a través de la RT 26}

Debemos comenzar el análisis de este punto numerando las normas técnicas que ponen en vigencia las normas internacionales de información financiera. Dichas normas son:

- Resolución Técnica $\mathrm{N}^{\circ} 26$

- Resolución Técnica $\mathrm{N}^{\circ} 27$ 
Caminando hacia una profesión globalizada. El proceso de adopción de las Normas ...

- Resolución 562/09 Comisión Nacional de Valores (adoptó la RT 26)

- Resolución 576/10 Comisión Nacional de Valores

- Resolución Técnica N 29 (modificó la RT 26 con nuevos puntos de Res. CNV 576/10)

- Circular de adopción NIIF $N^{\circ} 1$

- Circular de adopción NIIF $\mathrm{N}^{\circ} 2$

- Circular de adopción NIIF N³

\section{Antecedentes}

En 2007 el Directorio de la Comisión Nacional de Valores (ente regulador del mercado de capitales de Argentina) aprobó una propuesta elevada por la profesión para la adopción obligatoria de NIIF para entidades que cotizan en Bolsa, basada fundamentalmente en la experiencia de la Unión Europea. A partir de allí, la Mesa Directiva de FACPCE conformó una Comisión Especial para elaborar el Plan de Implementación de NIIF. En marzo de 2008, FACPCE presentó un borrador de la propuesta de conformación de esta Comisión a la CNV. A partir de este momento, comenzó la difusión del proyecto en eventos de la profesión, entre académicos contables y público en general.

Se recibieron numerosas observaciones y propuestas, las cuáles generaron la necesidad de elaborar un Plan Modificado, el cual fu presentado a CNV el 10 de octubre de 2008. Se procedió, además, a llevar adelante reuniones con el Directorio de este ente institucional para explicar detalles de la implementación del Plan y reuniones con autoridades provenientes de las contralorías provinciales a efectos de obtener un consenso para aplicación optativa por parte de todos los entes.

CENCyA, como ente motor de las normas contables profesionales aprobó el 22 de octubre de 2008 el Proyecto de Resolución Técnica y la Mesa Directiva FACPCE aprobó este proyecto el 28 de noviembre de 2008, fijando como periodo de consulta para todos los matriculados hasta el 01 de marzo de 2009. Una Comisión Especial analizó comentarios y sugerencias y remitió a CENCyA un "proyecto mejorado", el cuál fue aprobado por CENCyA el 05 de marzo de 2009. Finalmente, la Junta de Gobierno de FACPCE aprobó la RT 26 el 20 de marzo de 2009. 


\section{Consecuencias primarias que implica la adopción de estándares NIIF}

Concretamente, esta adopción presenta como consecuencias inmediatas:

- Obligatoriedad de utilizar NIIF para cierto tipo de entes, como se ve en el punto 5.3

- Opción de utilizarlas para otros

- Inclusión de las NIIF para pymes como opción, resultado de la modificación que la RT 29 efectuó para incluir las novedades generadas por la Resolución No 576/10 de la Comisión Nacional de Valores

- Modificación Reglamento General del CECyT, para contemplar la posibilidad de emisión de Circulares de Adopción de NIIF que pongan en vigencia las modificaciones o nuevas NIIF que se emitan en el futuro

- Necesidad de medidas de capacitación por parte de FACPCE, orientado a formar una masa crítica de contadores especializados en NIIF

- Definición de qué es una NIIF, entendiéndose a tales como aquellas generadas por el IASB en la versión oficial en español emitida por la IFRS Foundation, cuyo listado y fecha de última revisión se presenta en los Anexos I y II de RT 26. Incluye IAS (NIC), IFRS (NIIF), SIC, IFRIC e IFRS para SME (NIIF para pymes)

\section{Alcance y aplicación}

Para el análisis del alcance debemos identificar entre aquellos entes que deben utilizar NIIF completas en forma obligatoria de aquellos que pueden adoptarlas en forma voluntaria. Dentro del primer grupo se encuentran todas las entidades incluidas régimen de oferta pública de Ley No 17811, por su capital o por obligaciones negociables, o que hayan solicitado autorización para estar incluidas. Están exceptuadas de ello aquellas que cumplan con la definición anterior pero que tengan criterios contables particulares y distintos aceptados como tales por la 
Caminando hacia una profesión globalizada. El proceso de adopción de las Normas ...

Comisión Nacional de Valores, entre otros, los bancos, entidades de seguros, cooperativas y asociaciones civiles. Están alcanzadas además por la excepción, los entes que califican como Pyme de acuerdo a normas de SEPYME (Secretaría de la Pequeña y Mediana Empresa dependiente del Gobierno Nacional) y que cotizan en el régimen simplificado o en regímenes particulares de las bolsas del país.

Se encuentran, además, fuera de la aplicación obligatoria, los entes que estén incluidos en el Panel de Pymes que no realicen oferta pública y las restantes entidades bajo control de Comisión Nacional de Valores, tales como fondos comunes de inversión, fideicomisos financieros, mercados de futuros y opciones, bolsas de comercio y cajas de valores.

No obstante esta obligatoriedad, la norma prevé que el resto de los entes no incluidos en la aplicación obligatoria pueden optar por aplicar NIIF completas, la NIIF para pymes (sólo en el caso que estén incluidas en el concepto que el alcance de esta norma define para "pymes") o norma contable local, emitidas por la FACPCE (al día de hoy, el cuerpo ordenado de Resoluciones Técnicas).

\section{¿Hay posibilidad de dejar de aplicar NIIF?}

Para este análisis debemos distinguir entre los entes que aplican NIIF full y NIIF para pymes. Y dentro de los primeros, entre aquellos que aplican la norma por obligatoriedad de aquellos que la aplican por opción.

- Entes que aplican NIIF full por obligatoriedad: sólo podrán dejar de aplicar NIIF full en el caso que cesen las condiciones por las cuáles se encontraban obligados a hacerlo (genéricamente, si cesa la oferta pública de acciones u obligaciones negociables).

- Entes que aplican NIIF full por opción: sólo podrán dejar de aplicarlas cuando por razones debidamente fundadas se decidiera optar por NIIF para pymes o norma contable local. No se ha especificado ninguna definición para "razones debidamente fundadas", ni siquiera ejemplos, por lo que entendemos que queda a criterio del profesional. 
- Entes que aplican NIIF para pymes por opción: en razón de que la aplicación de esta norma sólo es optativa y limitada a aquellos entes que encuadren dentro del alcance de pyme que define la norma, puede dejar de aplicarse sólo cuando razones debidamente fundadas impidieran su aplicación.

- En los casos de cambio de norma, deberían aplicarse:

- Para los casos en que se aplicará norma contable local: los principios sobre modificación de ejercicios anteriores que surgen de la RT 8.

- Para los casos en que se aplicará NIIF full: si bien la norma no define ningún tratamiento, por analogía consideramos que debería aplicarse la NIC 8, específicamente en lo referido a cambios en las políticas contables.

\section{Aplicación integral}

Antes de proceder al análisis sobre las disposiciones sobre aplicabilidad integral o no de las NIIF, debemos considerar que en Argentina es aplicable la teoría del propietario, por la cual la participación de accionistas minoritarios sobre el grupo económico se presenta en un párrafo separado, entre el pasivo y el patrimonio neto. No es considerado pasivo por cuanto no existe ninguna obligación del grupo respecto de los mismos y no es considerado patrimonio neto porque no forma parte del patrimonio del accionista mayoritario. Esta teoría es opuesta a la que reviste las normas internacionales sobre participación en negocios conjuntos, que es la teoría del ente, por la cual todo el grupo económico es considerado un solo ente y la participación minoritaria incluida en el patrimonio neto.

La norma determina que para los casos de estados financieros individuales de entes que presentan sólo estados individuales (que no consolidan) y para los estados financieros consolidados, la aplicación de las NIIF es integral y sin ninguna modificación.

No obstante, se plantea una excepción para el caso de los estados contables individuales de los entes que deben presentar además el consolidado. En este caso, la aplicación es integral, excepto para la medición de la participación de la controlante en el patrimonio de la controlada en el estado financiero individual (separado) de la 
controlante, la cual no se valúa de acuerdo al párrafo 38 de la NIC 27 , sino a valor patrimonial proporcional, de acuerdo a RT 21 y NIC 28.

¿Qué significa esto? El párrafo 38 de la NIC 27 plantea que la medición de las inversiones en subsidiarias, controladas conjuntamente o sobre las que se ejerza influencia significativa, debe realizarse bajo el criterio de "costo o valor razonable, el menor". Por su parte, el método del valor patrimonial proporcional o "método de la participación" consiste básicamente en aplicar el porcentaje de participación accionaria sobre el patrimonio de la controlada y, en su caso, sobre los resultados. En virtud de la aplicación de la teoría del propietario, utilizar este último criterio es más conveniente en cuanto se persigue el objetivo de que el patrimonio y los resultados correspondientes a la participación controladora que surjan de los estados financieros consolidados presentados conjuntamente con estados financieros separados (individuales) sean iguales en ambos juegos de estados financieros.

Respecto de entidades que apliquen NIIF para pymes, la excepción a la aplicación integral es similar. En este caso, los entes que ejerzan control, control conjunto o influencia significativa, deberán aplicar, para medir sus participaciones en entes controlados, el "método de la participación”, según surge de la sección 14 Párrafo 14.8 de la NIIF para pymes.

\section{¿Cómo adopta la profesión argentina las modificaciones a NIIF o las nuevas NIIF emitidas en el futuro?}

Si partimos de la definición general de NIIF que determina la RT 26 , vemos que resultarían obligatorias las versiones oficiales del IASB en español. Lógicamente, existe un lapso de tiempo entre la emisión de la norma nueva o de una modificación en inglés y la emisión de la traducción oficial del IASB. Durante ese lapso, la FACPCE emitirá una versión en español, que será la que se encuentre vigente hasta la aparición de la versión IASB.

Las "Circulares de Adopción de NIIF" son las normas técnicas emitidas por el CENCyA que proponen la adopción de las nuevas 
versiones o normas internacionales por parte de los Consejos Profesionales. En estas circulares se incluye un breve resumen de las principales modificaciones y el contenido de la norma completa. Cabe destacar que, previamente a su emisión, cuando las circulares se encuentran en un periodo de consulta, las observaciones aceptadas son aquellas que hacen referencia a cuestiones de aplicación de la norma, como por ejemplo oposición a normas legales, no así en lo referido a aspectos técnicos, con lo cual se adoptan las normas tal cual fueron emitidas por el IASB.

\section{Fecha de vigencia y fecha de transición}

La aplicación de NIIF en forma obligatoria u opcional es a partir de ejercicios contables iniciados el 1 de enero de 2012, permitiéndose la aplicación anticipada.

La transición desde norma contable argentina a NIIF se desarrollará de acuerdo con las normas de la NIIF 1 y a las propias normas de transición que define la RT 26.

Esta norma requiere la consideración de la "fecha de transición", entendiéndose por tal a la fecha de inicio del periodo más antiguo en los estados financieros que el ente presentará información 100\% NIIF. Por ejemplo, si el primer cierre de ejercicio bajo NIIF es el 31 de diciembre de 2012, la fecha de transición sería el 31 de diciembre de 2010, como vemos en el siguiente gráfico.

Esta "fecha de transición" adquiere relevancia en cuanto la norma requiere que a partir de esa fecha comiencen a presentarse como información complementaria ciertas revelaciones y conciliaciones sobre los elementos de los estados financieros medidos bajo norma contable local y bajo NIIF. Todos estos elementos adquieren real relevancia cuando al cierre del primer periodo NIIF se deba presentar el estado de situación financiera con tres columnas (al 31/12/2010 -fecha de transición-, al 31/12/2011 y al 31/12/2012).

Algunos ejemplos de los requisitos de información que deben ser expuestos, de acuerdo con lo dispuesto por RT 26 (teniendo en cuenta como fecha de cierre del primer ejercicio bajo NIIF el 31/12/2012) son: 


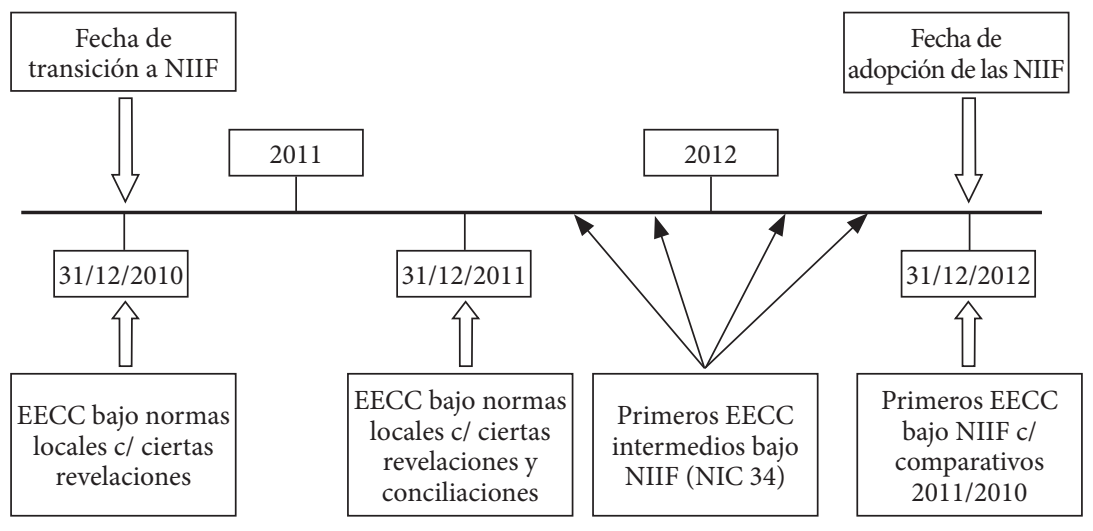

Fuente: elaboración propia

\begin{tabular}{|c|c|c|}
\hline $\begin{array}{l}\text { Requerimientos en } \\
\text { información complementaria }\end{array}$ & $\begin{array}{l}\text { Ejercicio } \\
2010\end{array}$ & $\begin{array}{c}\text { Ejercicio } \\
2011\end{array}$ \\
\hline $\begin{array}{l}\text { Identificar adopción de las NIIF, según RT } 26 \\
\text { y su puesta en vigencia (primer ejercicio anual } \\
\text { y trimestral aplicable) }\end{array}$ & $\mathrm{X}$ & \\
\hline $\begin{array}{l}\text { Expresar que se está considerando el impacto en } \\
\text { patrimonio }\end{array}$ & \multirow{2}{*}{$\mathrm{X}$} & \\
\hline $\begin{array}{l}\text { Expresar que se está evaluando el impacto en los } \\
\text { resultados }\end{array}$ & & \\
\hline $\begin{array}{l}\text { Conciliación del Patrimonio Neto según NIIF } \\
\text { y según normas FACPCE (extracontable) }\end{array}$ & $\mathrm{X}$ (opcional) & $\mathrm{X}$ \\
\hline NIIF que se aplicarán en el primer ejercicio bajo NIIF & $\mathrm{X}$ (opcional) & $\mathrm{X}$ \\
\hline $\begin{array}{l}\text { Conciliación del Patrimonio Neto según NIIF } \\
\text { y NCP al ejercicio anterior a NIIF }\end{array}$ & & $\mathrm{X}$ \\
\hline $\begin{array}{l}\text { Conciliación de Resultados según NIIF y según } \\
\text { normas FACPCE }\end{array}$ & & $\mathrm{X}$ \\
\hline $\begin{array}{l}\text { Conciliación entre efectivo y equivalente } \\
\text { (extracontable) }\end{array}$ & & $\mathrm{X}$ \\
\hline Cambios en NIIF utilizadas en conciliaciones & & $\mathrm{X}$ \\
\hline
\end{tabular}

Fuente: elaboración propia. 
Para el primer ejercicio cerrado anual con NIIF, se deberán tener en cuenta algunas consideraciones de importancia:

- Los asientos extracontables efectuados al 31/12/2010 y al 31/12/2011 para realizar la conciliación de las mediciones patrimoniales y de otros resultados integrales (al 31/12/2011) serán los primeros asientos del primer ejercicio bajo NIIF

- Se deberá presentar conciliación entre patrimonio medido con norma contable local y con NIIF a fecha de transición y a fecha anterior

- Además, se deberá incluir conciliación entre el resultado integral total medido de acuerdo con norma contable local y de acuerdo con NIIF

- Si los ajustes y reclasificaciones afectan al efectivo o al equivalente de efectivo, se debe presentar conciliación entre la medición con norma contable local y con NIIF

\section{Conclusiones}

El cambio de paradigma contable, símbolo de nuestros tiempos, hacia una contabilidad orientada a los mercados, trae como consecuencia la necesidad de ver al mundo como un único mercado de negocios. En este marco, contar con un lenguaje común facilitará la comparabilidad y el análisis de información contable por parte de inversores actuales y potenciales.

El actual proceso de convergencia llevado adelante entre el FASB y el IASB supone, en el mediano plazo, una revisión sistemática de las normas emitidas por cada uno de ellos y la consecución de que los nuevos estándares se estructuren alrededor de principios convergentes. A largo plazo podemos esperar la posibilidad de contar con un único juego de estándares globales (los Global GAAPs).

En este marco, la profesión argentina no quedó ajena a todo el proceso que el mundo estaba llevando adelante y a través de una adopción moderada (primero a través de una adaptación o convergencia y luego a través de la adopción) se insertó definitivamente en el universo NIIF.

El mapa de la normativa contable queda entonces definido de la siguiente manera: 
Caminando hacia una profesión globalizada. El proceso de adopción de las Normas ...

- NIIF full: aplicables en forma obligatoria para entes que cotizan, optativas para el resto

- NIIF para pymes: aplicable en forma optativa para entes no obligados a NIIF full, siempre que se encuentren dentro del alcance de pyme que define la norma

- Norma contable local (RTs): aplicable al resto de los entes, quienes no apliquen NIIF full en forma obligatoria ni NIIF para pymes por opción

De esta forma queda estructurada, por lo menos en el corto plazo, el esquema normativo. La tendencia se ajustará en función a la acogida que tengan las nuevas normas en los entes que las apliquen en forma obligatoria y de su difusión. No obstante ello, debemos destacar que se encuentran actualmente en estudio resoluciones de adopción de NIIF por parte de bancos, entidades financieras y entes de seguros.

Podemos finalizar este artículo haciendo uso de una frase que el IASB utiliza al presentar la adopción que están teniendo sus estándares en el mundo: "El mundo se está volviendo pequeño".

\section{Bibliografía}

Borges Quintanilla, Humberto. (2009). Convergencia en Latinoamérica IFRS-NIIF. Buenos Aires: La Ley.

Casinelli, Hernán. (2010). NIIF para las pymes. Buenos Aires: Aplicación Tributaria S.A.

(2008). Normas Internacionales de Información Financiera. Buenos Aires: Aplicación Tributaria S.A.

Departamento de Contabilidad de la USACH. (2009). IFRS-NIIF: casos de aplicación práctica. Chile: Thompson Reuters.

IFRS Foundation/Federación Argentina de Consejos Profesionales de Ciencias Económicas. (2012). Normas Internacionales de Información Financiera 2011. Buenos Aires: FACPCE.

IFRS Foundation/Federación Argentina de Consejos Profesionales de Ciencias Económicas. (2011). Normas Internacionales de Información Financiera para pymes 2009. Buenos Aires: FACPCE. 


\section{Juan Pablo Romano Pastor}

cpn_jpromanopastor@yahoo.com.ar

Contador Público Nacional (Universidad del Aconcagua - Argentina). Docente e investigador de la misma universidad. Tiene posgrado en Contabilidad Superior y Normas Internacionales de Información Financiera (Universidad Nacional de Cuyo). Sus temas de interés han girado en torno a la ética profesional, las normas internacionales de contabilidad, el control interno, entre otros temas disciplinares y profesionales. 